\title{
Conformal anomaly of super Wilson loop
}

\author{
A.V. BELITSKY \\ Department of Physics, Arizona State University \\ Tempe, AZ 85287-1504, USA
}

\begin{abstract}
Classically supersymmetric Wilson loop on a null polygonal contour possesses all symmetries required to match it onto non-MHV amplitudes in maximally supersymmetric Yang-Mills theory. However, to define it quantum mechanically, one is forced to regularize it since perturbative loop diagrams are not well-defined due to presence of ultraviolet divergences stemming from integration in the vicinity of the cusps. A regularization that is adopted by practitioners by allowing one to use spinor helicity formalism, on the one hand, and systematically go to higher orders of perturbation theory is based on a version of dimensional regularization, known as Four-Dimensional Helicity scheme. Recently it was demonstrated that its use for the super Wilson loop at one loop breaks both conformal symmetry and Poincaré supersymmetry. Presently, we exhibit the origin for these effects and demonstrate how one can undo this breaking. The phenomenon is alike the one emerging in renormalization group mixing of conformal operators in conformal theories when one uses dimensional regularization. The rotation matrix to the diagonal basis is found by means of computing the anomaly in the Ward identity for the conformal boost. Presently, we apply this ideology to the super Wilson loop. We compute the one-loop conformal anomaly for the super Wilson loop and find that the anomaly depends on its Grassmann coordinates. By subtracting this anomalous contribution from the super Wilson loop we restore its interpretation as a dual description for reduced non-MHV amplitudes which are expressed in terms of superconformal invariants.
\end{abstract}




\section{Introduction}

In the past decade, the planar maximally supersymmetric Yang-Mills (SYM) theory became a laboratory for developing and testing various techniques for strong coupling analysis of fourdimensional gauge theories via the AdS/CFT correspondence. These discoveries stretch far beyond consequences of the exact superconformal symmetry of the theory valid to any loop order [1]. The focus of the last several years was the space-time S-matrix of the regularized theoryt

Due to the fact that all propagating fields of the $\mathcal{N}=4$ SYM can be combined into a single light-cone superfield $\Phi$ [1, 2], the $n$-particle matrix element of the S-matrix can be organized in a single superamplitude $\mathcal{A}_{n}$ which is defined in turn by the amputated Green function $\left\langle\Phi_{1} \Phi_{2} \ldots \Phi_{n}\right\rangle_{\mathrm{amp}}$. Making use of the translation invariance and Poincaré supersymmetry, one can extract the (super)momentum conservation laws from the superamplitude and write it in the following generic form [2]

$$
\mathcal{A}_{n}=i(2 \pi)^{4} \frac{\delta^{(4)}\left(\sum_{i} \lambda_{i} \tilde{\lambda}_{i}\right) \delta^{(8)}\left(\sum_{i} \lambda_{i} \eta_{i}\right)}{\langle 12\rangle\langle 23\rangle\langle 34\rangle \ldots\langle n-1 n\rangle} \widehat{\mathcal{A}}_{n}\left(\lambda_{i}, \widetilde{\lambda}_{i}, \eta_{i} ; a\right) .
$$

Here we introduced a notation for the 't Hooft coupling constant $a=g^{2} N_{c} /\left(4 \pi^{2}\right)$ and used the spinor helicity formalism to represent the particles' momenta $p_{i}^{\dot{\alpha} \alpha}=\widetilde{\lambda}^{\dot{\alpha}} \lambda^{\alpha}$ and chiral charges $q_{\alpha}^{A}=\eta^{A} \lambda_{\alpha}$ in terms of commuting Weyl spinors $\lambda_{\alpha}$ and anticommuting Grassmann variables $\eta^{A}$. Here the angle brackets are conventionally defined as $\langle i j\rangle=\lambda_{i}^{\alpha} \lambda_{j \alpha}$. The dependence of the scattering amplitude on particle helicities involved in scattering emerges from the expansion of the reduced superamplitude $\widehat{\mathcal{A}}_{n}$ in terms of $\eta$ 's. Namely, it admits the following series

$$
\widehat{\mathcal{A}}_{n}=\widehat{\mathcal{A}}_{n, 0}+\widehat{\mathcal{A}}_{n, 1}+\ldots,
$$

with each term being a homogeneous polynomial of degree $\eta^{4 k}$. This expansion terminates at the term of order $k=n-4$ due to nilpotence of Grassmann variables and superconformal symmetry. The leading term of the reduced amplitude starts with unity at zero order in coupling since the maximal helicity-violating (MHV) amplitude was extracted from $\mathcal{A}_{n}$ in the form of the Parke-Taylor tree formula [3]. The subleading terms are identified with $\mathrm{N}^{k} \mathrm{MHV}$ amplitudes, $\widehat{\mathcal{A}}_{n, k}=\widehat{\mathcal{A}}_{n}^{\mathrm{N}^{k} \mathrm{MHV}}$. While the tree superamplitude enjoys the full superconformal symmetry of the theory, one violates a number of them as quantum effects are taken into account, such that the dilatation, conformal boost and superconformal transformations are broken [4] by the infrared diverges due to copious emissions of particles.

As was discovered more recently [5], if the tree superamplitude is rewritten in terms of the socalled region supermomenta $\left(x_{i}, \theta_{i}^{A}\right)$, which solve automatically the supermomentum conservation conditions in Eq. (1),

$$
\widetilde{\lambda}_{i}^{\dot{\alpha}} \lambda_{i}^{\alpha}=x_{i i+1}^{\dot{\alpha} \alpha}, \quad \lambda_{i}^{\alpha} \eta_{i}^{A}=\theta_{i i+1}^{\alpha A},
$$

the former exhibits yet another symmetry. The latter is the so-called dual superconformal symmetry which acts on $\left(x_{i}, \theta_{i}^{A}\right)$ as if they were coordinates (not momenta). Making use of these coordinates, the tree-level NMHV amplitudes can be written in terms of superconformal invariants as follows [5]

$$
\widehat{\mathcal{A}}_{n, 1}=\sum_{1<q<r<n} R_{n q r}
$$

\footnotetext{
${ }^{1}$ Since the theory does not develop a mass gap, the four-dimensional scattering amplitudes vanish due to infrared divergences intrinsic to theories with massless gauge bosons.
} 
where

$$
R_{p q r}=-\frac{\langle q-1 q\rangle\langle t-1 t\rangle \delta^{(4)}\left(\Xi_{p q r}\right)}{x_{q r}^{2}\left\langle r\left|x_{p r} x_{r q}\right| q-1\right\rangle\left\langle p\left|x_{p r} x_{r q}\right| q\right\rangle\left\langle p\left|x_{p q} x_{q r}\right| r-1\right\rangle\left\langle p\left|x_{p q} x_{q r}\right| r\right\rangle},
$$

and the argument of the Grassmann delta function is given by the expression

$$
\Xi_{p q r}=\sum_{i=r}^{p-1} \eta_{i}\left\langle i\left|x_{r q} x_{q p}\right| p\right\rangle+\sum_{i=p}^{q-1} \eta_{i}\left\langle i\left|x_{q r} x_{r p}\right| p\right\rangle \text {. }
$$

Similar representations were found for all tree $\mathrm{N}^{k} \mathrm{MHV}$ amplitudes [6].

Analogously to the ordinary superconformal symmetry, some of the dual superconformal generators become anomalous at loop level [5]. The explicit realization of the dual superconformal symmetry was suggested in terms of super Wilson loops [7, 8] that admit the following generic form 9 ]

$$
\left\langle\mathcal{W}_{n}\left(x_{i}, \theta_{i} ; a\right)\right\rangle=\frac{1}{N_{c}}\left\langle\operatorname{tr} P \exp \left(\frac{1}{2} i g \int_{\mathcal{C}_{n}} d x^{\dot{\alpha} \alpha} \mathcal{A}_{\alpha \dot{\alpha}}+i g \int_{\mathcal{C}_{n}} d \theta^{\alpha A} \mathcal{F}_{\alpha A}\right)\right\rangle,
$$

in terms of the bosonic $\mathcal{A}_{\alpha \dot{\alpha}}$ and fermionic $\mathcal{F}_{\alpha A}$ connections that are power series in the Grassmann variables $\theta$ with $x$-dependent coefficients being the propagating fields of the $\mathcal{N}=4$ SYM theory. The super Wilson loop is stretched on a contour $\mathcal{C}_{n}$ in superspace formed by segments connecting the vertices located at $\left(x_{i}, \theta_{i}\right)$ that are connected by straight lines. This is a generalization of an earlier conjecture for the dual representation for the MHV amplitudes [10, 11, 12, 13. obtained from above by setting all Grassmann variables to zero. The former is well tested by now confronting it against multi-loop/multileg results on the amplitude side [14, 15, 16, 17, 18, 19, 20]. In the latter case, the bosonic Wilson loop has a polygonal contour $C=\left[x_{1}, x_{2}\right] \cup\left[x_{2}, x_{3}\right] \ldots\left[x_{n}, x_{1}\right]$ with its sites defined by particle momenta $p_{i}=x_{i i+1}$. The dual superconformal symmetry of superamplitudes is realized as ordinary superconformalsymmetry of the super Wilson loop. The precise correspondence between the latter and non-MHV amplitudes is purpoted to work as follows. The expansion of $\mathcal{W}_{n}$ in terms of fermionic variables goes in powers of $\theta^{4}$

$$
\left\langle\mathcal{W}_{n}\right\rangle=\left\langle\mathcal{W}_{n ; 0}\right\rangle+\left\langle\mathcal{W}_{n ; 1}\right\rangle+\ldots
$$

with $\left\langle\mathcal{W}_{n ; k}\right\rangle$ being a homogeneous polynomial of order $4 k$, and formally can go all the way to $\theta^{4 n}$. Then the fulfillment of the duality between the two objects requires

$$
\left\langle\mathcal{W}_{n ; k}\left(x_{i}, \theta_{i} ; a\right)\right\rangle=a^{k} \widehat{\mathcal{A}}_{n ; k}\left(\lambda_{i}, \widetilde{\lambda}_{i}, \eta_{i} ; a\right),
$$

and all components with $k>n-4$ vanishing. However, while this correspondence was verified for the lowest component $\left\langle\mathcal{W}_{n ; 0}\right\rangle$ by explicit strong [10] and weak [11, 12, 13] coupling analyses that confirmed the duality for MHV amplitudes, the foundation of the above matching for super Wilson loop in Minkowski space with non-MHV amplitudes faced difficulties and requires further elucidation. Namely, the consideration performed in Ref. [21] demonstrated that due to on-shell nature of the closure of the supersymmetry algebra in maximally supersymmetric Yang-Mills theory and highly singular nature of superloop on light-like polygonal contour, the Poincaré supersymmetry gets broken already at one loop order by effects stemming from the field equations of motion when one uses a version of dimensional regularization known as Four-Dimensional Helicity scheme [22]. The same anomalous contribution violates conformal symmetry thus invalidating the naive duality between the superamplitude and super Wilson loop. 
However, as was already pointed out in Ref. [21], the supersymmetric Wilson loop is a scheme dependent quantity inheriting the property from its purely bosonic progenitor. The very fact of the scheme dependence of the super Wilson loop can be verified by applying the operator product expansion (OPE) reasoning to the multipoint correlation functions of the stress-tensor supermultiplet $\mathcal{T}$. These were identified as yet another equivalent representation of non-MHV scattering amplitudes in Refs. [23] and checked in trees and loops. Again this generalized the conjecture valid for the MHV case [24]. As a consequence the resolution of the above puzzle is hiding in the fact that only the product of the OPE coefficient function and the vacuum expectation value of the superloop is well-defined. Thus one may reshuffle the anomalous contributions away from the latter into the former. This is alike a finite scheme transformation. Similar phenomena are not new and were studied previously in other circumstances as we will discuss below.

Since the discussion parallels the light-cone conformal OPE for two-point functions, let us discuss it first since we will acquire from it the intuition on the scheme-dependent pattern of conformal symmetry breaking. It suffices to limit the consideration to the leading-twist, i.e., twist two, contribution to the OPE for the product of two dimension- $d_{\mathcal{O}}$ protected operators as $x^{2} \rightarrow 0$

$$
\mathcal{O}(x) \mathcal{O}(0)=\frac{1}{\left(x^{2}\right)^{d_{\mathcal{O}}-1}} \sum_{j} C_{j}\left(\mu^{2} x^{2} ; a\right) \mathbb{O}_{j}\left(0 ; \mu^{2}\right)+\ldots
$$

where the ellipses stand for contributions of higher twist operators. The sum in the right-hand side runs over the conformal spin $j$ of contributing operators of canonical dimension $d_{j}$. The coefficient function $C_{j}$ admits an infinite series expansion in 't Hooft coupling $a$ and depends on the dimensionless product of the near light-like distance $x^{2}$ and the factorization scale $\mu^{2}$. The twist-two conformal operators $\mathbb{O}_{j}$ develop corresponding dependence on the renormalization scale $\mu^{2}$ due to their nonvanishing anomalous dimensions $\gamma_{j}(a)$. The above expansion separates the physics of short and long light-cone distances, encoding the former/latter into the coefficient function/conformal operator. In writing above OPE, one tacitly assumes that the conformal covariance is preserved by the perturbative scheme adopted in the analysis of ultraviolet divergences in the renormalization of conformal operators $\mathbb{O}_{j}$, such that the latter obey autonomous renormalization group equations

$$
\frac{d}{d \log \mu} \mathbb{O}_{j}=-\gamma_{j}(a) \mathbb{O}_{j}
$$

and as a consequence, the coefficient function can be factorized into a perturbatively corrected Clebsh-Gordan coefficient in the decomposition of the product of two dimension- $d_{\mathcal{O}}$ representation into irreducible components which is expressed in terms of the confluent hypergeometric function [25]

$$
C_{j}\left(\mu^{2} x^{2} ; a\right)=c_{j}(a)\left(\mu^{2} x^{2}\right)_{1}^{\gamma_{j}(a)} F_{1}\left(\frac{1}{2}\left(d_{j}+j+\gamma_{j}(a)+1\right), d_{j}+j+\gamma_{j}(a)+1 ; x \cdot \partial\right)
$$

and a coupling-dependent coefficient $c_{j}(a)$.

It is important to realize that while the left-hand side of the (10) is independent of the factorization scale $\mu$ and is scheme independent, the OPE introduces ultraviolet divergences in individual terms and thus dependence on the way they are treated perturbatively for both conformal operators and coefficient functions. This scheme dependence is intrinsic to the anomalous dimensions of conformal operators. Namely, using conventional regularization procedures based on a departing from four space-time dimensions, be it the original dimensional regularization 
or dimensional reduction etc., and the (modified) minimal subtraction scheme, one immediately discovers that conformal operators mix with each other starting from two-loop order,

$$
\frac{d}{d \log \mu} \mathbb{O}_{j}^{\mathrm{MS}}=-\sum_{k \leq j} \gamma_{j k}(a) \mathbb{O}_{k}^{\mathrm{MS}}
$$

with $\gamma_{j>k}(a) \sim a^{2}$. This phenomenon was well studied in QCD [26]. In this theory, the reason for mixing is two-fold. First, the conformal symmetry gets broken by the renormalization of the QCD strong coupling which induces non-trivial beta function, $\beta \neq 0$. Second, there is another effect, present in any theory, conformal or not, which is more subtle. This is the effect that we are after here being interested in application to $\mathcal{N}=4$ theory. Whenever dimensional regularization is used the conformal invariance is broken even if the four-dimensional beta function $\beta$ vanishes since the coupling becomes dimensional away from four dimensions $g \rightarrow g_{\varepsilon}=g \mu^{\varepsilon}$ and therefore $\beta_{\varepsilon}=-\varepsilon g$. This $O(\varepsilon)$ effect when accompanied by $1 / \varepsilon$ poles in perturbative graphs generates finite, anomalous contributions. The studies of conformal Ward identities for the correlation function with conformal operator insertion allowed one to compute a (finite) scheme transformation matrix $\mathcal{B}$ to the conformally covariant basis of operators obeying Eq. (11),

$$
\mathbb{O}_{j}^{\mathrm{MS}}=\sum_{k \leq j} \mathcal{B}_{j k} \mathbb{O}_{k}
$$

Here the matrix $\mathcal{B}$ is determined by the special conformal anomaly matrix $\gamma_{j k}^{c}$ as follows $\mathcal{B}_{j k}=$ $-a_{j k}^{-1} \sum_{l<j} \gamma_{j l}^{c} \mathcal{B}_{l k}$ and arises from the renormalization of the product of the operator $\mathbb{O}_{j}^{\mathrm{MS}}$ and conformal boost variation of the regularized action

$$
\mathbb{O}_{j}^{\mathrm{MS}}\left(\delta_{\kappa} S\right)=\sum_{k \leq j} \gamma_{j k}^{c}(a) \mathbb{O}_{k}^{\mathrm{MS}}
$$

Thus $\mathcal{B}$ diagonalizes the mixing matrix $\gamma_{j k}$ by removing from it conformal symmetry breaking effects.

Now, let us turn to the correlation functions of the stress-tensor supermultiplet $\mathcal{T}$ [23],

$$
G_{n}\left(x_{i}, \theta_{i} ; a\right)=\left\langle\mathcal{T}\left(x_{1}, \theta_{1}\right) \ldots \mathcal{T}\left(x_{n}, \theta_{n}\right)\right\rangle
$$

with anti-chiral Grassmann variables set to zero. Recently, it demonstrated found that their multiple pairwise light-cone limit $x_{i i+1}^{2} \rightarrow 0$ is related to the square of the full superamplitude $23]$

$$
\lim _{x_{i i+1}^{2} \rightarrow 0} G_{n}\left(x_{i}, \theta_{i} ; a\right) \sim\left(\prod_{i=1}^{n} x_{i i+1}^{-2}\right)\left(\sum_{k=0}^{n-4} a^{k} \widehat{\mathcal{A}}_{n, k}\left(\lambda_{i}, \widetilde{\lambda}_{i}, \eta_{i} ; a\right)\right)^{2} .
$$

It is important to realize that the correlation function is well-defined in four dimensions away from the light-cone limit and thus does not require a regularization. However, by taking the limit, one effectively constructs an OPE similar to the previously discussed two-point case. Then the leading term in the expansion is given by the product of the (square of the) vacuum expectation value of the super Wilson loop and a coefficient function,

$$
\lim _{x_{i i+1}^{2} \rightarrow 0} G_{n}\left(x_{i}, \theta_{i}\right)=\left(\prod_{i=1}^{n} x_{i i+1}^{-2}\right) C\left(g ; \mu^{2} x_{i j}^{2}, \theta_{i}\right)\left\langle\mathcal{W}_{n}\left(x_{i}, \theta_{i}\right)\right\rangle^{2} .
$$


The former, being an eikonal approximation for particle's propagation, encodes the long-distance physics. On the other hand, the latter takes care of the rest. Therefore, while the product of the two is a scheme independent observable, each of them separately does depend on the on the way to handle the divergences. The Wilson loop is singular even away from the lightcone limit due to the mere presence of the cusps on its contour be it lights like or not. This is pertinent to the purely bosonic case where the identification of the scattering amplitudes with the expectation value of the Wilson loop on a light-light polygonal contour requires a specific choice for identification of the infrared scale parameter on the amplitude side with the ultraviolet one, on the other. This scheme dependence becomes trickier as one ascends to superspace. Though the final result of one-loop calculations of the super Wilson loop corresponding to NMHV level of scattering amplitudes is finite, the intermediate steps require a regularization procedure. What this implies is that the latter breaks the conformal symmetry at the intermediate steps and this effect can permeate into the final answer as an anomaly. This is one-to-one correspondence with the argument alluded to above for the conformal operators. The difference is however that the anomaly contributes starting already from one loop which is a consequence of the more singular nature of the light-like Wilson loop: have the one-loop renormalization of conformal operators induce a double pole in $\varepsilon$, the anomalous dimension matrix of the latter would be off-diagonal already at one loop, not two.

The desire to use helicity formalism for scattered particles severely constraints the choice of acceptable regularization schemes. Since one conventionally uses a variation of dimensional regularization for calculations of scattering amplitudes which allows one, on the one hand, to preserve supersymmetry and, on the other, straightforward generalization beyond one-loop order, it is paramount to adopt the same scheme for perturbative analysis of dual observables. As we advocated earlier, the use of a version of dimensional regularization, known as FDH scheme, which yields anomalous contributions in Wilson loop expectation values thus spoiling the duality between them and the non-MHV scattering amplitudes. Therefore, in order to restore it, one has to subtracting the anomalous terms computing the latter from conformal Ward identities. By an explicit calculation, we will demonstrate below that at one loop in the Four-Dimensional Helicity scheme

$$
a \widehat{\mathcal{A}}_{n ; 1}=\left\langle\mathcal{W}_{n ; 1}\right\rangle-a_{n ; 1},
$$

where $a_{n ; 1}$ is the anomalous contribution arising from breaking of the special conformal boost by the regularization procedure at loop level. Notice that due to the specific origin of ultraviolet divergences in the super Wilson loop as arising from the light-like nature of the polygon sides connecting nearest pairs of vertices, only the graphs were the virtual particles are exchanged (at one loop) between the nearest and next-to-nearest links require regularization. All other contributions, involving exchanges separated by more than one site, are finite. This implies that since each link carries two Grassmann variables associated with them, the anomalies will be present in only for NMHV amplitudes with four adjacent Grassmann variables.

Our subsequent presentation is organized as follows. In the next section, we remind the definition of the super Wilson loop along with conventions used. In Section 2, we perform oneloop computations of the $\left\langle\mathcal{W}_{n, 1}\right\rangle$ component of the super Wilson loop for $n=5,6$ to support the structure suggested by Eq. (19). Then in Section 3, we construct conformal Ward identities for the super Wilson loop and calculate one-loop conformal anomaly for all tree-level NMHV amplitudes. We demonstrate that indeed it explains the anomalous nature of the loop and necessitates a finite subtraction to restore conformal symmetry. Finally, we comment on our results. 


\section{Superymmetric Wilson loop at one loop}

The supersymmetric Wilson loop that we presently study is defined by the path-ordered product of the links $\mathcal{W}_{[i i+1]}$

$$
\left\langle\mathcal{W}_{n}\right\rangle=\frac{1}{N_{c}}\left\langle\operatorname{tr}\left(\mathcal{W}_{[12]} \ldots \mathcal{W}_{[n 1]}\right)\right\rangle
$$

on segments of the polygonal contour $\mathcal{C}_{n}$ that are parametrized as

$$
x_{[i i+1]}=x_{i}-t x_{i i+1}, \quad \theta_{[i i+1]}=\theta_{i}-t \theta_{i i+1} .
$$

The individual superlines are exponents of the line integrals of superconnections

$$
\mathcal{W}_{[i i+1]}=P \mathrm{e}^{i g E_{[i i+1]}}
$$

with $E_{[i i+1]}$ admitting an expansion in Grassmann variables

$$
E_{[i i+1]}=\sum_{n} E_{[i i+1]}^{[n]}
$$

with each term in the sum $E_{[i i+1]}^{[n]}$ being a polynomial of order $n$ in $\theta$ 's. They read explicitly (up to the fourth order)

$$
\begin{aligned}
& E_{[i i+1]}^{[0]}=-\frac{1}{2} \int_{0}^{1} d t\langle i|A| i] \\
& E_{[i i+1]}^{[1]}=-\frac{i}{2} \chi_{i}^{A} \int_{0}^{1} d t\left[\bar{\psi}_{A} i\right] \\
& E_{[i i+1]}^{[2]}=-\frac{i}{2} \chi_{i}^{A} \int_{0}^{1} d t\left(\frac{1}{2}\left\langle\theta_{[i i+1]}^{B}|D| i\right]+\eta_{i}^{B}\right) \bar{\phi}_{A B}, \\
& E_{[i i+1]}^{[3]}=-\frac{1}{3} \varepsilon_{A B C D} \chi_{i}^{A} \int_{0}^{1} d t\left(\frac{1}{4}\left\langle\theta_{[i i+1]}^{B}|D| i\right]+\eta_{i}^{B}\right)\left\langle\theta_{[i i+1]}^{C} \psi^{D}\right\rangle, \\
& E_{[i i+1]}^{[4]}=-\frac{i}{8} \varepsilon_{A B C D} \chi_{i}^{A} \int_{0}^{1} d t\left(\frac{1}{6}\left\langle\theta_{[i i+1]}^{B}|D| i\right]+\eta_{i}^{B}\right)\left\langle\theta_{[i i+1]}^{C}|F| \theta_{[i i+1]}^{D}\right\rangle .
\end{aligned}
$$

Here, we introduced new notations so a few comments are in order. First, we wrote the above relations in terms of the fermionic components $\chi_{i}^{A}$ of momentum supertwistors [27] related to the Grassmann coordinates of the contour as $\chi_{i}^{A}=\left\langle i \theta_{i}^{A}\right\rangle$. We use the bra and ket formalism to write down the inner products, such that by defining $\left\langle A\left|=A^{\alpha},\right| A\right\rangle_{\alpha},\left[A \mid=A_{\dot{\alpha}}\right.$ and $\left.\mid A\right]=A^{\dot{\alpha}}$ we have $\langle A B\rangle=A^{\alpha} B_{\alpha}$ and $[A B]=A_{\dot{\alpha}} B^{\dot{\alpha}}$. Then inverting the relation for $\chi_{i}^{A}$ in favor of fermionic coordinates of the cusps $\theta_{i \alpha}^{A}$, one finds

$$
\left|\theta_{i}^{A}\right\rangle=\frac{\chi_{i-1}^{A}|i\rangle-\chi_{i}^{A}|i-1\rangle}{\langle i-1 i\rangle}
$$

This immediately provides a relation of $\chi$ 's to the Grassmann variables on the superamplitude side,

$$
\left|\theta_{i i+i}^{A}\right\rangle=\eta_{i}^{A}|i\rangle, \quad \eta_{i}^{A}=\frac{\chi_{i-1}^{A}}{\langle i-1 i\rangle}+\frac{\chi_{i+1}^{A}}{\langle i i+1\rangle}-\frac{\langle i-1 i+1\rangle}{\langle i-1 i\rangle\langle i i+1\rangle} \chi_{i}^{A} .
$$


Next, each term in the line integral over the superconnections goes over the fields populating the $\mathcal{N}=4$ SYM supermuliplet, the gauge field $A_{\alpha \dot{\alpha}}$, the gaugino $\bar{\psi}_{\dot{\alpha} A}$, the scalars $\phi_{A B}$ in the $\mathbf{6}$ of $\mathrm{SU}(4)$, the conjugate gaugino $\psi^{\alpha A}$ and the chiral component of the gluon field-strength tensor $F^{\alpha \beta}=\frac{1}{4}\left(\sigma_{\mu \nu}\right)^{\alpha \beta} F^{\mu \nu}$. The covariant derivatives $D_{\alpha \dot{\alpha}}=\partial_{\alpha \dot{\alpha}}-i g\left[A_{\alpha \dot{\alpha}},\right]$ in the above equations act on the argument $x_{[i i+1]}$ of the fields only. Finally, the subleading terms that we do not display in the expansion in Eq. (23), which goes up to $\theta^{8}$, are either interaction-dependent and thus vanish in the free-field limit or proportional to the equation of motion. Since in the present paper we will be after the $\mathcal{W}_{n ; 1}$ term, none of these will be of relevance for our analysis.

The one-loop calculation of super Wilson loop was already performed in Ref. [21] for four points. Contrary to the expectation based on the conjectured duality between the amplitudes and supersymmetric Wilson loop, the found result was nonvanishing. That calculation was done making use the standard FDH regularization scheme especially well adopted for helicity formalism used in handling gauge theory amplitudes. The discovered result was purely anomalous. It was simultaneously breaking Poincaré supersymmetry and special conformal boosts. However, the consistency of the regularization procedure was demonstrated by derivation of supersymmetric Ward identities and an independent calculation of arising supersymmetric anomalies. Below, extending earlier considerations [21, we will demonstrate that the anomalous contribution to super Wilson loop can be isolated and subtracted out in the fashion that we advocated in the Introduction.

In order to understand the structure of the result, without obscuring it with unnecessary details, we will focus in this section only on the $\chi_{1}^{4}$ contribution 2 to the supersymmetric Wilson loop expectation value. The conjectured duality claims that it should be equal to tree gluon NMHV amplitude multiplied by the factor of the 't Hooft couping, more precisely to $\chi_{1}^{4}$ component of the sum of $R$-superinvariant defining it at tree level (4). Extracting the component in question from the result of our earlier work [21], we find

$$
\left\langle\mathcal{W}_{4 ; 1}\right\rangle=-\frac{a}{48} \frac{[42]}{[41][12]} \frac{\langle 42\rangle^{3}}{\langle 41\rangle^{3}\langle 12\rangle^{3}} \chi_{1}^{4}+\ldots
$$

Below we compute one-loop contribution to the same component for the pentagon and hexagon and determine the common pattern resulting from it. As a consequence we will be able to generalize our consideration to any number of points.

\subsection{Pentagon}

First, let us begin with the pentagon. At one-loop order, the contribution to the component in question arises from just two components of the superconnections, such that the expectation value is given by the following correlation function

$$
\left\langle\mathcal{W}_{5 ; 1}\right\rangle=\frac{(i g)^{2}}{N_{c}} \sum_{i}\left\langle\operatorname{tr}\left(E_{[12]}^{[4]} E_{[i i+1]}^{[0]}\right)\right\rangle,
$$

where $E^{[0]}$ and $E^{[4]}$ are determined by the first and last equation in (24), respectively. This function receives nontrivial contributions from the vertex and exchange diagrams, displayed in

\footnotetext{
${ }^{2}$ Here and everywhere in the paper, we introduce a shorthand notation $\chi_{i} \chi_{j} \chi_{k} \chi_{l} \equiv \varepsilon_{A B C D} \chi_{i}^{A} \chi_{j}^{B} \chi_{k}^{C} \chi_{l}^{D}$ to simplify presentation of formulas.
} 


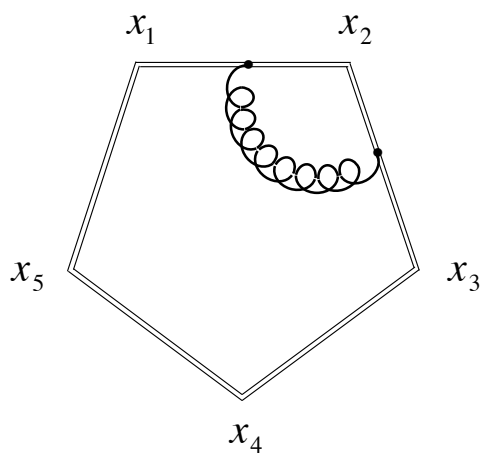

(a)

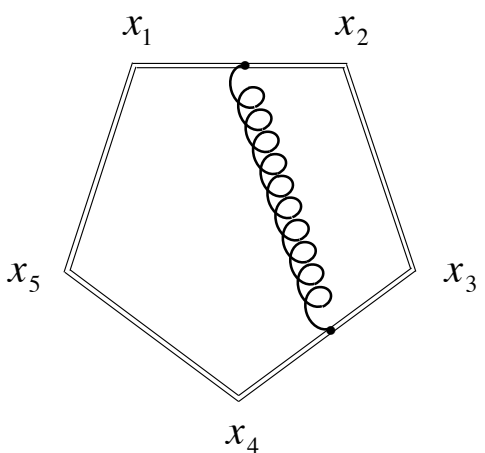

(b)

Figure 1: One-loop contribution to $\chi_{1}^{4}$ component of the pentagon supersymmetric Wilson loop.

Fig. 1 (a) and (b), respectively, and their mirror reflections $(\bar{a})$ and $(\bar{b})$. As we already advertised in the Introduction, in order to perform the analysis efficiently we will use the rules of the FDH regularization scheme [22]. Its main advantage is that while being a version of dimensional regularization, it allows one to perform the spinor decomposition of supermomenta even in regularized theory and rely on four-dimensional manipulation rules like Fierz transformation etc. The propagator entering the computation reads

$$
\left\langle A_{\alpha \dot{\alpha}}\left(x_{1}\right) F_{\gamma \delta}\left(x_{2}\right)\right\rangle=i \frac{\Gamma(2-\varepsilon)}{4 \pi^{2-\varepsilon}} \frac{1}{\left[-x_{12}^{2}\right]^{2-\varepsilon}}\left(\varepsilon_{\alpha \delta}\left(x_{12}\right)_{\dot{\alpha} \beta}+\varepsilon_{\alpha \beta}\left(x_{12}\right)_{\dot{\alpha} \delta}\right) .
$$

Notice that individual graphs induce logarithmic terms depending on Mandelstam invariants which cancel only in the sum of 1 (b) and its mirror $(\bar{b})$. We will not display them in intermediate formulas that follow. The individual contributions read respectively for the graphs in Fig. 1 (a) and its mirror

$$
\left\langle\mathcal{W}_{5 ; 1}^{(\mathrm{a})+(\overline{\mathrm{a}})}\right\rangle=-\frac{a}{48} \frac{\langle 52\rangle^{2} \chi_{1}^{4}}{\langle 51\rangle^{2}\langle 12\rangle^{2}}\left\{\frac{1}{x_{13}^{2}}+\frac{1}{x_{52}^{2}}\right\}
$$

as well as diagrams (b) and its mirror $(\bar{b})$

$$
\begin{aligned}
& \left\langle\mathcal{W}_{5 ; 1}^{(\mathrm{b})}\right\rangle=-\frac{a}{48} \frac{\chi_{1}^{4}}{\langle 51\rangle^{3}\langle 12\rangle^{2}} \frac{\left\langle 5\left|x_{53}\right| 3\right]}{x_{13}^{2}\left(x_{14}^{2}-x_{24}^{2}\right)}\left\{\langle 13\rangle\langle 52\rangle^{2}-\langle 23\rangle\left\langle 5\left|x_{63}\right| 3\right]\left(\frac{\langle 12\rangle\langle 35\rangle}{x_{14}^{2}}-\frac{\langle 23\rangle\langle 51\rangle}{x_{24}^{2}}\right)\right\}, \\
& \left\langle\mathcal{W}_{5 ; 1}^{(\overline{\mathrm{b}})}\right\rangle=-\frac{a}{48} \frac{\chi_{1}^{4}}{\langle 51\rangle^{2}\langle 12\rangle^{3}} \frac{\left\langle 2\left|x_{42}\right| 4\right]}{x_{52}^{2}\left(x_{42}^{2}-x_{41}^{2}\right)}\left\{\langle 41\rangle\langle 52\rangle^{2}-\langle 45\rangle\left\langle 2\left|x_{42}\right| 4\right]\left(\frac{\langle 51\rangle\langle 24\rangle}{x_{42}^{2}}-\frac{\langle 12\rangle\langle 45\rangle}{x_{41}^{2}}\right)\right\} .
\end{aligned}
$$

Making use of the identity

$$
\langle 12\rangle\langle 13\rangle \frac{\left\langle 5\left|x_{53}\right| 3\right]}{x_{13}^{2}}-\langle 51\rangle\langle 41\rangle \frac{\left\langle 2\left|x_{42}\right| 4\right]}{x_{24}^{2}}=-\left(x_{14}^{2}-x_{24}^{2}\right) \frac{x_{35}^{2}}{[51][12]},
$$

it allows one to rewrite the sum of the last two contributions in a form free from the spurious poles at $x_{14}^{2}=x_{24}^{2}$, such that it can be cast in the form

$$
\left\langle\mathcal{W}_{5 ; 1}^{(\mathrm{b})+(\overline{\mathrm{b}})}\right\rangle=\frac{a}{48} \frac{\langle 52\rangle^{2} \chi_{1}^{4}}{\langle 51\rangle^{2}\langle 12\rangle^{2}} \frac{x_{35}^{2}}{x_{13}^{2} x_{52}^{2}}\left\{1+\frac{[34]^{2}}{x_{24}^{2} x_{41}^{2}} \frac{\langle 23\rangle^{2}\langle 45\rangle^{2}}{\langle 52\rangle^{2}}\right\} .
$$


Adding all these terms together and using the identity $x_{35}^{2}=x_{13}^{2}+x_{52}^{2}-\langle 52\rangle[52]$, one finds that the pentagon Wilson loop reads

$$
\left\langle\mathcal{W}_{5 ; 1}\right\rangle=-\frac{a}{48} \chi_{1}^{4}\left\{\frac{[52]}{[51][12]} \frac{\langle 52\rangle^{3}}{\langle 51\rangle^{3}\langle 12\rangle^{3}}-\frac{x_{35}^{2}[34]^{2}}{x_{13}^{2} x_{24}^{2} x_{41}^{2} x_{52}^{2}} \frac{\langle 23\rangle^{2}\langle 45\rangle^{2}}{\langle 51\rangle^{2}\langle 12\rangle^{2}}\right\} .
$$

A naked eye inspection immediately reveals that the first term in the curly brackets is identical (making use of the obvious replacement $4 \rightarrow 5$ since now the point 5 becomes adjacent to 1 ) to the anomalous contribution defining the four-cusp Wilson loop quoted in the preamble to this section. Then the remaining term has to be identical to the five-point reduced NMHV amplitude $\widehat{\mathcal{A}}_{5 ; 1}$. Indeed, for five points the NMHV amplitude is nothing else but the $\overline{\mathrm{MHV}}$, i.e, conjugate to MHV. However, for subsequent generalization to more points it is instructive to recall its expression in terms of superconformal invariants. Namely, the tree five-particles NMHV amplitude is simply determined by one $R$-invariant and reads [28]

$$
\widehat{\mathcal{A}}_{5,1}=R_{241} \text {. }
$$

The complicated general form of the latter simplifies enormously for five points and takes the following well-known form

$$
R_{241}=\delta^{(4)}\left(\eta_{1}[23]+\eta_{2}[13]+\eta_{3}[21]\right) \frac{\langle 12\rangle\langle 23\rangle\langle 34\rangle\langle 45\rangle\langle 51\rangle}{\langle 45\rangle^{4}[12][23][34][45][51]} .
$$

Cyclic invariance of the amplitude implies that $R_{241}=R_{413}$ upon the use of the (super)momentum conserving delta-functions, which provides an alternative but identical representation. Expanding the $R$-invariant in components one finds for the contribution in question

$$
R_{241}=-\frac{x_{35}^{2}[34]^{2}}{x_{13}^{2} x_{24}^{2} x_{41}^{2} x_{52}^{2}} \frac{\langle 23\rangle^{2}\langle 45\rangle^{2}}{\langle 51\rangle^{2}\langle 12\rangle^{2}} \chi_{1}^{4}+\ldots
$$

It is thus readily identified with the second term in the curly brackets of Eq. (32).

\subsection{Hexagon and up}

Now we address a less trivial example which involves a genuine NMHV amplitude, the hexagon. The nonvanishing Feynman diagrams that define the component in question are shown in Fig. 2. As for the pentagon case, we will display only rational contribution from each of the graph, ignoring the logarithmic terms which cancel in the total sum. The calculations are straightforward for Fig. 2 (a) and its mirror and yield

$$
\left\langle\mathcal{W}_{6 ; 1}^{(\mathrm{a})+(\overline{\mathrm{a}})}\right\rangle=-\frac{a}{48} \frac{\chi_{1}^{4}\langle 62\rangle^{2}}{\langle 61\rangle^{2}\langle 12\rangle^{2}}\left\{\frac{1}{x_{13}^{2}}+\frac{1}{x_{62}^{2}}\right\},
$$

as well as for Fig. 2 (b) and its mirror, respectively, one finds

$$
\begin{aligned}
& \left\langle\mathcal{W}_{6 ; 1}^{(\mathrm{b})}\right\rangle=-\frac{a}{48} \frac{\chi_{1}^{4}}{\langle 61\rangle^{3}\langle 12\rangle^{2}} \frac{\left\langle 6\left|x_{63}\right| 3\right]}{x_{13}^{2}\left(x_{14}^{2}-x_{24}^{2}\right)}\left\{\langle 13\rangle\langle 62\rangle^{2}-\langle 23\rangle\left\langle 6\left|x_{63}\right| 3\right]\left(\frac{\langle 12\rangle\langle 36\rangle}{x_{14}^{2}}-\frac{\langle 23\rangle\langle 61\rangle}{x_{24}^{2}}\right)\right\}, \\
& \left\langle\mathcal{W}_{6 ; 1}^{(\overline{\mathrm{b}})}\right\rangle=-\frac{a}{48} \frac{\chi_{1}^{4}}{\langle 61\rangle^{2}\langle 12\rangle^{3}} \frac{\left\langle 2\left|x_{52}\right| 5\right]}{x_{62}^{2}\left(x_{52}^{2}-x_{51}^{2}\right)}\left\{\langle 51\rangle\langle 62\rangle^{2}-\langle 56\rangle\left\langle 2\left|x_{52}\right| 5\right]\left(\frac{\langle 61\rangle\langle 25\rangle}{x_{52}^{2}}-\frac{\langle 12\rangle\langle 56\rangle}{x_{51}^{2}}\right)\right\} .
\end{aligned}
$$




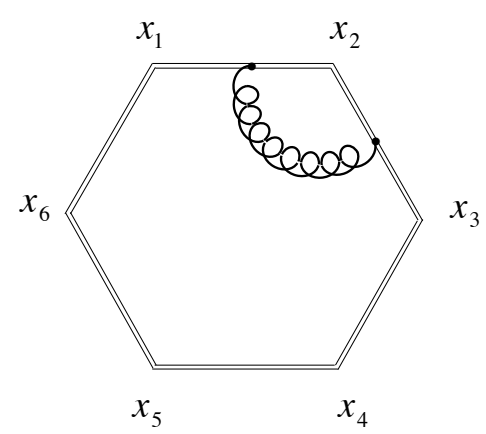

(a)

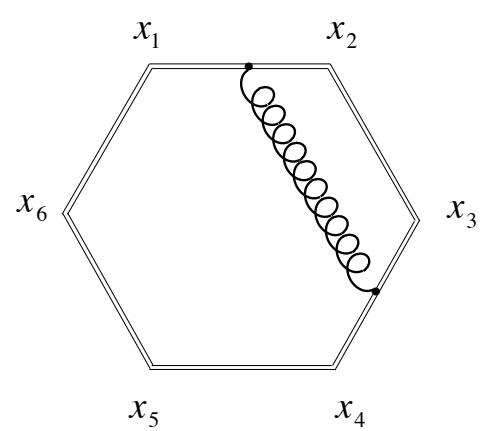

(b)

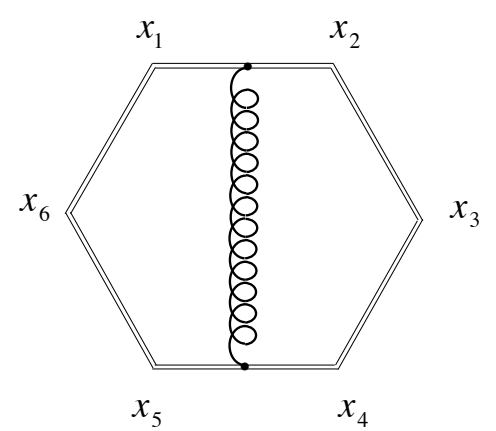

(c)

Figure 2: Non-vanishing one-loop $\chi_{1}^{4}$-contribution to the hexagon Wilson loop.

The exchange diagram in Fig. 2 (c) require a more tedious analysis and multiple use of identities for angle and square brackets to finally bring the result to a concise form

$$
\begin{aligned}
& \left\langle\mathcal{W}_{6 ; 1}^{(\mathrm{c})}\right\rangle=\frac{a}{48} \frac{\chi_{1}^{4}}{\langle 61\rangle^{3}\langle 12\rangle^{3}}\left\{\langle 14\rangle\langle 62\rangle^{2} \frac{\langle 23\rangle\langle 61\rangle[34]-\langle 12\rangle\langle 56\rangle[45]}{\left(x_{14}^{2}-x_{24}^{2}\right)\left(x_{52}^{2}-x_{51}^{2}\right)}\right. \\
& +\frac{\left\langle 1\left|x_{14}\right| 4\right]}{x_{14}^{2} x_{52}^{2}-x_{24}^{2} x_{51}^{2}}\left[\frac{\langle 23\rangle^{2}\left\langle 6\left|x_{63}\right| 3\right]^{2}}{x_{14}^{2}-x_{25}^{2}}\left(\frac{\langle 12\rangle\langle 46\rangle}{x_{14}^{2}}-\frac{\langle 61\rangle\langle 24\rangle}{x_{24}^{2}}\right)\right. \\
& \left.\left.+\frac{\langle 56\rangle^{2}\left\langle 2\left|x_{52}\right| 5\right]^{2}}{x_{52}^{2}-x_{51}^{2}}\left(\frac{\langle 12\rangle\langle 46\rangle}{x_{52}^{2}}-\frac{\langle 61\rangle\langle 24\rangle}{x_{51}^{2}}\right)\right]\right\} .
\end{aligned}
$$

Notice that for uniformity of presentation, the numerator in the first line can be further rewritten in the form mimicking the rest of the result $\langle 23\rangle\langle 61\rangle[34]-\langle 12\rangle\langle 56\rangle[45]=\langle 62\rangle\left[4\left|x_{41}\right| 1\right\rangle+$ $\langle 61\rangle\langle 12\rangle[14]$ and use the identity $\langle 14\rangle[14]=x_{14}^{2}-x_{24}^{2}-x_{51}^{2}+x_{52}^{2}$. While the denominator in second line is a "square" $x_{14}^{2} x_{52}^{2}-x_{24}^{2} x_{51}^{2}=\left\langle 1\left|x_{14}\right| 4\right]\left\langle 4\left|x_{14}\right| 1\right]$. After a lengthy calculation, one finds for the sum of all terms

$$
\begin{aligned}
\left\langle\mathcal{W}_{6 ; 1}\right\rangle & =-\frac{a}{48} \chi_{1}^{4}\left\{\frac{[62]}{[61][12]} \frac{\langle 62\rangle^{3}}{\langle 61\rangle^{3}\langle 12\rangle^{3}}\right. \\
& \left.+\frac{\langle 23\rangle\langle 34\rangle\langle 45\rangle\langle 56\rangle}{\langle 61\rangle^{3}\langle 12\rangle^{3}\left\langle 4\left|x_{14}\right| 1\right]}\left[\frac{\left\langle 6\left|x_{63}\right| 3\right]^{3}}{\langle 45\rangle\langle 56\rangle[12][23] x_{14}^{2}}-\frac{\left\langle 6\left|x_{52}\right| 3\right]^{3}}{\langle 23\rangle\langle 34\rangle[56][61] x_{52}^{2}}\right]\right\} .
\end{aligned}
$$

Again, the first term is the anomalous contribution, which has the same universal form as for the four- and five-point Wilson loops, while the second term is a known two-term representation of the NMHV amplitude [29] obtained by solving the BCFW recursion relations [30]. It can be re-written in terms of the $\chi_{1}^{4}$ component of the sum of the $R$-invariants defining the six-particle NMHV amplitude $R_{146}+R_{136}+R_{135}$ [5. The latter equivalent to the three-term form of the gluon scattering amplitude originally computed in Ref. [31].

As a consequence of this analysis, one can immediately generalize the consideration to more points without the need to go through explicit computations. Namely, the $\chi_{1}^{4}$ component of the loop Wilson loop is given by a sum of the universal anomalous contribution and the corresponding Grassmann component of the $R$-invariant defining the NMHV scattering amplitude,

$$
\left\langle\mathcal{W}_{n ; 1}\right\rangle=-\frac{a}{48} \chi_{1}^{4}\left\{\frac{[n 2]}{[n 1][12]} \frac{\langle n 2\rangle^{3}}{\langle n 1\rangle^{3}\langle 12\rangle^{3}}+\left.\sum_{1<q<r<n} R_{n q r}\right|_{\chi_{1}^{4}}\right\} .
$$


Let us now turn to understanding the source of the anomalous term.

\section{Conformal anomaly}

In the previous sections, we observed by explicit calculations that the $\chi_{1}^{4}$ component of the super Wilson loop computed making use of the FDH scheme develops universal anomalous contributions. The variation of this component of the super Wilson loop can be computed explicitly using the conformal variation of its components, and reads

$$
\delta\left\langle\mathcal{W}_{n ; 1}\right\rangle=-\frac{a}{48} \chi_{1}^{4} \frac{\langle n 2\rangle^{2}}{\langle n 1\rangle^{2}\langle 12\rangle^{2}}\left[\frac{1}{x_{n 2}^{2}}\left(3\langle n|\kappa| n]-\langle 1|\kappa| n] \frac{\langle n 2\rangle}{\langle 12\rangle}\right)-\frac{1}{x_{13}^{2}}\left(3\langle 2|\kappa| 2]-\langle 1|\kappa| 2] \frac{\langle n 2\rangle}{\langle n 1\rangle}\right)\right] .
$$

In the present section we will derive this result along with all other Grassmann degree 4 contributions to the conformal anomaly of the supersymmetric Wilson loop. To this end we derive conformal Ward identities for the latter following the approach developed in Refs. [26] and recently used for the bosonic Wilson loop [5] in order to fix the conformal symmetry-breaking part of the MHV amplitudes.

Writing the expectation value of superloop as a path integral

$$
\left\langle\mathcal{W}_{n}\right\rangle=\int[D X] \mathcal{W}_{n} \mathrm{e}^{i S[X]}
$$

with the integration performed over all fields propagating fields and ghosts, cumulatively called $X$. Then using the invariance of the path integrals under the conformally transformed field variables, one can easily derive the Ward identity

$$
\delta_{\kappa}\left\langle\mathcal{W}_{n}\right\rangle=\left\langle\mathcal{W}_{n}\left(i \delta_{\kappa} S\right)\right\rangle
$$

where vacuum average on the right-had side involves the non-vanishing conformal variation of the regularized action. The latter is of the form

$$
\left(i \delta_{\kappa} S\right)=-4 i \varepsilon \int d^{D} z(\kappa \cdot z) \Delta(z)+\ldots
$$

with

$$
\Delta(z)=\frac{1}{2}\left[F^{\alpha \beta} F_{\alpha \beta}+\bar{F}_{\dot{\alpha} \dot{\beta}} \bar{F}^{\dot{\alpha} \dot{\beta}}\right]+\frac{i}{2}\left[\bar{\psi}_{\dot{\alpha}}\left(D^{\dot{\alpha} \alpha} \psi_{\alpha}\right)-\left(D_{\alpha \dot{\alpha}} \bar{\psi}^{\dot{\alpha}}\right) \psi^{\alpha}\right]+\frac{1}{4}\left(D_{\mu} \phi^{A B}\right)\left(D^{\mu} \bar{\phi}_{A B}\right),
$$

where the ellipses stand for neglected BRST exact operators which do not contribute to the gauge-invariant correlation functions that we are currently investigating. Notice also that we ignored in the right-hand side of Eq. (43) the vanishing term involving the conformal boost variation of the super Wilson loop $\delta_{\kappa} \mathcal{W}_{n}=0$.

The right-hand side of the Ward identity contains the anomalous term involving the conformal variation of the action which is expressed in terms of integrated operator insertion $\Delta(z)$ with

\footnotetext{
${ }^{3}$ Their explicit transformation properties are $\delta_{\kappa} \chi_{i}=0, \delta_{\kappa}\left(x_{i j}^{2}\right)^{\mp 1}= \pm 2\left(x_{i j}^{2}\right)^{-1} \kappa \cdot\left(x_{i}+x_{j}\right), \delta_{\kappa}\langle i j\rangle^{ \pm 1}=$ $\pm\langle i j\rangle^{-1}\left\langle i\left|\kappa x_{j}+x_{i} \kappa\right| j\right\rangle, \delta_{\kappa}\langle i i+1\rangle^{ \pm 1}= \pm 2\langle i i+1\rangle^{ \pm 1} \kappa \cdot x_{i+1}$.
} 


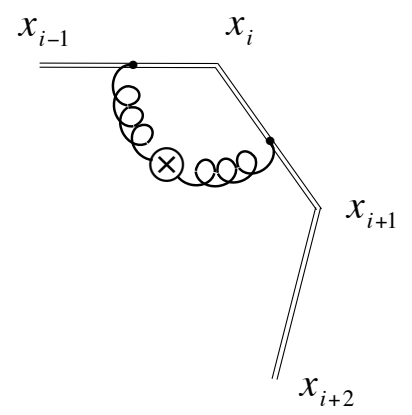

(a)

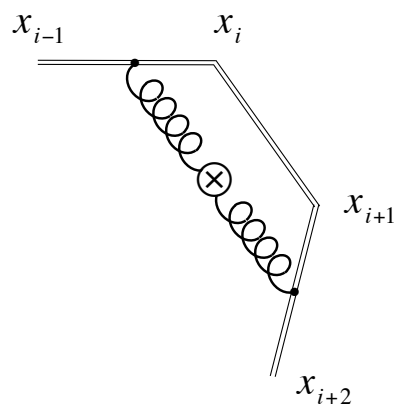

(b)

Figure 3: Feynman diagrams contributing to the conformal anomaly from gauge fields. The $\otimes$ stands for the insertion of the conformal variation of the action.

the coordinate weight $z$. In order to extract the contribution in question is proves convenient to introduce the Fourier transform of the resulting correlation function

$$
\widetilde{\mathcal{W}}_{n}(k)=\int d^{D} z \mathrm{e}^{i k \cdot z}\left\langle\mathcal{W}_{n} \Delta(z)\right\rangle
$$

and while calculating it we keep only terms linear in the momentum $k$, such that the anomaly on the right-hand side of the Ward identity coms from its second terms in the Taylor expansion

$$
\left\langle\mathcal{W}_{n}\left(i \delta_{\kappa} S\right)\right\rangle=-4 \varepsilon\left(\kappa \cdot \partial_{k}\right)_{k=0} \widetilde{\mathcal{W}}_{n}(k)
$$

Obviously, the anomaly admits the expansion in Grassmann variables

$$
\widetilde{\mathcal{W}}_{n}=\widetilde{\mathcal{W}}_{n ; 0}+\widetilde{\mathcal{W}}_{n ; 1}+\ldots,
$$

identical to the one for the superloop itself (8) .

\subsection{Bosonic Wilson loop revisited}

Let us start our analysis of the one-loop calculation by revisiting the purely bosonic case [5]. At one-loop, the anomaly $\widetilde{\mathcal{W}}_{n ; 0}$

$$
\widetilde{\mathcal{W}}_{n ; 0}=\sum_{i<j}^{n} A_{[i i+1][j j+1] ; 0}
$$

is given by the sum of the gluon exchanges between different sites

$$
A_{[i i+1][j j+1] ; 0}=\frac{(i g)^{2}}{N_{c}} \int d^{D} z \mathrm{e}^{i k \cdot z}\left\langle\operatorname{tr} E_{[i i+1]}^{[0]} E_{[j j+1]}^{[0]} \Delta^{\mathrm{g}}(z)\right\rangle,
$$

where $\Delta^{g}$ is given by the first term in Eq. (45) which is just the covariant gauge boson action $-\frac{1}{4} F_{\mu \nu}^{2}$. This reads in the single-gluon exchange approximation

$$
\begin{aligned}
A_{[i i+1][j j+1] ; 0} & =-\frac{g^{2} N_{c}}{2} \int_{0}^{1} d s x_{i i+1}^{\mu} \int_{0}^{1} d t x_{j j+1}^{\nu} \\
& \times \int \frac{d^{D} k_{1} d^{D} k_{2}}{(2 \pi)^{D}} \delta^{(D)}\left(k_{1}+k_{2}+k\right) \frac{\mathrm{e}^{-i k_{1} \cdot x_{[i i+1]}}}{k_{1}^{2}} \frac{\mathrm{e}^{-i k_{2} \cdot x_{[j j+1]}}}{k_{2}^{2}}\left(k_{1 \nu} k_{2 \mu}-g_{\mu \nu} k_{1} \cdot k_{2}\right) .
\end{aligned}
$$


Here we used the propagator in the Feynman gauge

$$
\left\langle A_{\alpha \dot{\alpha}}\left(x_{1}\right) A_{\beta \dot{\beta}}\left(x_{2}\right)\right\rangle=2 i \int \frac{d^{D} k}{(2 \pi)^{D}} \frac{\mathrm{e}^{-i k \cdot x_{12}}}{k^{2}} \varepsilon_{\alpha \beta} \varepsilon_{\dot{\alpha} \dot{\beta}} .
$$

In what follows, we will be extracting terms linear in the momentum $k$ only and thus all equation below should be understood modulo all other (irrelevant for us) contributions. Let us consider the interaction exchanges between the site [12] and the rest. First, a simple calculation immediately yields for the $1 / \varepsilon$-pole contribution arising from nearest-neighbor interaction displayed in Fig. 3 (a),

$$
A_{[12][23] ; 0}=-a \frac{\Gamma(1-\varepsilon)}{32 \varepsilon}\left(-\mu^{2} x_{13}^{2} \pi\right)^{\varepsilon}\left\{3\langle 1|k| 1]-3\langle 2|k| 2]-4 k \cdot x_{2}+\frac{8}{\varepsilon} k \cdot x_{2}\right\} .
$$

One observes the presence of translationally non-invariant contribution in the single-pole term. The latter has to cancel against additional Feynman diagrams. A quick inspection shows that the only other graph that also develops divergent contribution is the one where the gluon is exchanged between the next-to-nearest neighbor sites, see Fig. 3(b). This implies that all $\left.A_{[12][k k+1] ; 0}\right|_{3<k<n-1}$ are finite. One obtains after a straightforward calculation

$$
A_{[12][34] ; 0}=-a \frac{\Gamma(1-\varepsilon)}{16 \varepsilon}\left(-\mu^{2} x_{13}^{2} \pi\right)^{\varepsilon} k \cdot\left(x_{2}+x_{3}\right),
$$

which is not translationally invariant either. Adding the two, one finds

$$
A_{[12][23] ; 0}+A_{[12][34] ; 0}=-a \frac{\Gamma(1-\varepsilon)}{32 \varepsilon}\left(-\mu^{2} x_{13}^{2} \pi\right)^{\varepsilon}\left\{3\langle 1|k| 1]-4\langle 2|k| 2]+\frac{8}{\varepsilon} k \cdot x_{2}\right\},
$$

where the translational invariance of the single-pole contribution is restored. On the other hand, it appears that this finding is different from the result of Ref. [5]. The resolution of this puzzle merely lies in a different arrangement of terms in the single-pole contribution. Namely, adding mirror symmetric diagrams (with the symmetry axis going through the link-[12]) to the two graphs computed above, one can re-arrange terms in the sum of next-to-nearest neighbor graphs as

$$
\begin{aligned}
A_{[12][34] ; 0}+A_{[12][n-1 n] ; 0} & =-\frac{a}{16 \varepsilon}\left[k \cdot\left(x_{1}+x_{3}\right)+k \cdot\left(x_{n}+x_{2}\right)\right] \\
& \equiv \bar{A}_{[12][34] ; 0}+\bar{A}_{[12][n-1 n] ; 0}
\end{aligned}
$$

such that the sum of the first term in the square brackets with Eq. (153) gives the well-known form for the two-site conformal anomaly,

$$
A_{[12][23] ; 0}+\bar{A}_{[12][34] ; 0}=-a \frac{\Gamma(1-\varepsilon)}{32 \varepsilon}\left(-\mu^{2} x_{13}^{2} \pi\right)^{\varepsilon}\left\{4\langle 1|k| 1]-4\langle 2|k| 2]+\frac{8}{\varepsilon} k \cdot x_{2}\right\} .
$$

It is interesting to note that the diagram Fig. 3 (b) does not contribute to the calculation of Ref. [5]. This is achieved by means of a gauge transformation of the Wilson-loop links, such that each site acquires a total-derivative contribution. So effectively the authors of Ref. [5] do not use the Feynman gauge. Namely, the addition of total-derivative terms amounts to replacement of the integrand in Eq. (51) as follows,

$$
k_{1 \nu} k_{2 \mu}-g_{\mu \nu} k_{1} \cdot k_{2} \rightarrow k_{1 \nu} k_{2 \mu}-k_{1 \mu} k_{2 \nu}-g_{\mu \nu} k_{1} \cdot k_{2},
$$


i.e., $A_{[i i+1][j j+1] ; 0} \rightarrow A_{[i i+1][j j+1] ; 0}-G_{[i i+1][j j+1]}$. Note that the extra contribution $k_{1 \mu} k_{2 \nu}$ produces vanishing effect in a gauge-invariant Wilson loop. However, what it achieves locally is to shift the effect of next-to-nearest graphs to the vertex-type diagrams in Fig. 3 (a). The reason for this is that all graphs but the vertex-like become finite after the substitution (58) as can be seen from the explicit form of the extra term

$$
\begin{aligned}
& G_{[i i+1][j j+1]}=-a \frac{\Gamma(1-\varepsilon)}{16 \varepsilon}\left\{\left(-\mu^{2} x_{i+1 j+1}^{2}\right)^{\varepsilon} k \cdot\left(x_{i+1}+x_{j+1}\right)+\left(-\mu^{2} x_{i j}^{2}\right)^{\varepsilon} k \cdot\left(x_{i}+x_{j}\right)\right. \\
&\left.-\left(-\mu^{2} x_{i+1 j}^{2}\right)^{\varepsilon} k \cdot\left(x_{i+1}+x_{j}\right)-\left(-\mu^{2} x_{i j+1}^{2}\right)^{\varepsilon} k \cdot\left(x_{i}+x_{j+1}\right)\right\} .
\end{aligned}
$$

This is finite for $\left.G_{[12][k k+1]}\right|_{3<k<n-1}$, while $\operatorname{div}_{\varepsilon} A_{[12][34] ; 0}=\operatorname{div}_{\varepsilon} G_{[12][34]}$ (and similarly for mirror symmetric graph). It immediately yields the result in Eq. (57) when subtracted from $A_{[12][23] ; 0}$.

\subsection{Supersymmetric Wilson loop}

Now we are in a position to compute the one-loop anomaly to the Grassmann degree 4 contribution to the supersymmetric Wilson loop $\mathcal{W}_{n ; 1}$. We are not going to restrict ourselves to just the $\chi_{1}^{4}$-component, but consider $\mathcal{W}_{n ; 1}$ in full generality instead. This requires the inclusion of diagrams with scalars and fermions as well in addition to gauge fields. The only two types of graphs that diverge at one loop are the same ones displayed in Fig. 3 with the obvious replacement of gluon propagators by either scalars or gauginos. The conformal anomaly at one-loop order for the $\theta^{4}$ component will be then determined by the sum pairwise contributions, identical to the one in Eq. (49), where now $A=A^{\mathrm{g}}+A^{\mathrm{f}}+A^{\mathrm{s}}$. Let us discuss these in turn.

\subsubsection{Scalars}

Due to its simplicity, let us start with exchanges of scalars. Their link- $[i i+1]$ to link-[jj +1$]$ correlator receives contributions from $E^{[2]}$ 's in (24) and reads

$$
A_{[i i+1][j j+1] ; 1}^{\mathrm{s}}=\frac{(i g)^{2}}{N_{c}} \int d^{D} z \mathrm{e}^{i k \cdot z}\left\langle\operatorname{tr} E_{[i i+1]}^{[2]} E_{[j j+1]}^{[2]} \Delta^{\mathrm{s}}(z)\right\rangle,
$$

where the scalar insertion $\Delta^{\mathrm{s}}$ is given by the last term in Eq. (45). It is instructive to split the calculation into blocks and first compute the correlation function of $\Delta^{\mathrm{s}}$ with scalar fields. Extracting only the terms linear in $k$, one finds after taking the integral

$$
\Delta_{A B, C D}\left(x_{1}, x_{2}\right)=\int d^{D} z \mathrm{e}^{i k \cdot z}\left\langle\bar{\phi}_{A B}\left(x_{1}\right) \Delta_{\mathrm{s}}(z) \bar{\phi}_{C D}\left(x_{2}\right)\right\rangle=-\varepsilon_{A B C D} \frac{\Gamma(1-\varepsilon)}{8 \pi^{2-\varepsilon}} \frac{k \cdot\left(x_{1}+x_{2}\right)}{\left[-x_{12}^{2}\right]^{1-\varepsilon}},
$$

where the regularized coordinate-space propagators we used reads

$$
\left\langle\bar{\phi}_{A B}\left(x_{1}\right) \bar{\phi}_{C D}\left(x_{2}\right)\right\rangle=\frac{\Gamma(1-\varepsilon)}{4 \pi^{2-\varepsilon}} \frac{\varepsilon_{A B C D}}{\left[-x_{12}^{2}\right]^{1-\varepsilon}} .
$$

Next, inserting Eq. (61) into the contour of the supersymmetric Wilson loop, one gets after a lengthy computation for the pole part of the divergent Feynman graphs,

$$
\begin{aligned}
A_{[12][23] ; 1}^{\mathrm{s}} & =a \frac{\Gamma(1-\varepsilon)}{32 \varepsilon}\left(-x_{13}^{2} \mu^{2} \pi\right)^{\varepsilon} \frac{\chi_{1} \chi_{2}}{x_{13}^{2}\langle 12\rangle^{2}} \\
& \times\left\{\chi_{2}\left(\chi_{2}-\langle 12\rangle \eta_{1}\right)\langle 1|k| 2]-\chi_{1}\left(\chi_{1}-\langle 12\rangle \eta_{2}\right)\langle 2|k| 1]-\chi_{1} \chi_{2}(\langle 1|k| 1]-\langle 2|k| 2])\right\},
\end{aligned}
$$


and

$$
A_{[12][34] ; 1}^{\mathrm{s}}=a \frac{\Gamma(1-\varepsilon)}{32 \varepsilon}\left(-x_{13}^{2} \mu^{2} \pi\right)^{\varepsilon} \frac{\chi_{1} \chi_{2} \chi_{3}}{\langle 12\rangle\langle 23\rangle}\left\{\chi_{1} \frac{\langle 2|k| 1]}{x_{13}^{2}}-\chi_{3} \frac{\langle 2|k| 3]}{x_{24}^{2}}\right\},
$$

for the nearest- and next-to-nearest-neighbors, respectively.

\subsubsection{Gauginos}

Next, we turn to the contribution of fermions to the anomaly,

$$
A_{[i i+1][j j+1] ; 1}^{\mathrm{f}}=\frac{(i g)^{2}}{N_{c}} \operatorname{tr} \int d^{D} z \mathrm{e}^{i k \cdot z}\left\langle E_{[i i+1]}^{[3]} E_{[j j+1]}^{[1]} \Delta^{\mathrm{f}}(z)\right\rangle
$$

where the insertion $\Delta^{\mathrm{f}}$ is now determined by the second term in Eq. (45). Since this insertion is just the fermion equations of motion, the calculation can be easily done with propagators in the coordinate representation, i.e.,

$$
\left\langle\psi_{\alpha}\left(x_{1}\right) \bar{\psi}_{\dot{\alpha}}\left(x_{2}\right)\right\rangle=i \frac{\Gamma(2-\varepsilon)}{2 \pi^{2-\varepsilon}} \frac{\left(x_{12}\right)_{\alpha \dot{\alpha}}}{\left[-x_{12}^{2}\right]^{2-\varepsilon}},
$$

since the insertion $\Delta^{\mathrm{f}}$ is effectively moved onto the contour due to the contact nature of the emerging interaction,

$$
\partial_{1}^{\dot{\beta} \alpha}\left\langle\psi_{\alpha}\left(x_{1}\right) \bar{\psi}_{\dot{\alpha}}\left(x_{2}\right)\right\rangle=\delta_{\dot{\alpha}}^{\dot{\beta}} \delta^{(D)}\left(x_{12}\right),
$$

and thus leaving just one propagator. The operator insertion, expanded to linear order in the momentum $k$, takes the form

$$
\Delta_{\alpha \dot{\alpha}}{ }_{B}^{A}\left(x_{1}, x_{2}\right)=\int d^{D} z \mathrm{e}^{i k \cdot z}\left\langle\psi_{\alpha}^{A}\left(x_{1}\right) \Delta^{\mathrm{f}}(z) \bar{\psi}_{\dot{\alpha} B}\left(x_{2}\right)\right\rangle=-\frac{i \Gamma(2-\varepsilon)}{4 \pi^{2-\varepsilon}} k \cdot\left(x_{1}+x_{2}\right) \frac{x_{12 \alpha \dot{\alpha}}}{\left[-x_{12}^{2}\right]^{2-\varepsilon}} \delta^{A}{ }_{B} .
$$

Then a calculation of the diagram displayed in Fig. 3 (a) with the gauge boson line being replaced by the fermion one yields

$$
A_{[12][23] ; 1}^{\mathrm{f}}=a \frac{\Gamma(1-\varepsilon)}{48 \varepsilon}\left(-x_{13}^{2} \mu^{2} \pi\right)^{\varepsilon} \frac{\chi_{1}^{2} \chi_{2}}{x_{13}^{2}\langle 12\rangle^{2}}\left\{\chi_{1}\langle 2|k| 1]+\chi_{2}\langle 1|k| 1]-\langle 2|k| 2]\left(2 \chi_{2}-\langle 12\rangle \eta_{1}\right)\right\} .
$$

Finally, the exchange diagram of the type given in Fig. 3 (b) produces

$$
A_{[12][34] ; 1}^{\mathrm{f}}=a \frac{\Gamma(1-\varepsilon)}{48 \varepsilon}\left(-x_{13}^{2} \mu^{2} \pi\right)^{\varepsilon} \frac{\chi_{1} \chi_{2} \chi_{3}}{\langle 12\rangle\langle 23\rangle}\left\{\chi_{2} \frac{\langle 3|k| 3]}{x_{24}^{2}}-\chi_{1} \frac{\langle 2|k| 1]}{x_{13}^{2}}\right\} .
$$

The contribution with interchanged Grassmann degrees on the sites, i.e., $\left\langle E_{[i i+1]}^{[1]} E_{[j j+1]}^{[3]} \Delta^{\mathrm{f}}(z)\right\rangle$ is determined from the above making use of the substitution $1 \leftrightarrow 2$ and flipping the overall sign. Notice that both results are independently translationally invariant as the shift-breaking terms, present at the intermediate steps, cancel between contributions of the bosonic and fermionic connections to the individual Feynman graphs and serve as cross-check on the calculation. 


\subsubsection{Gauge fields}

Finally, we come to the gauge fields which are computationally the most involved contributions. The analysis is best performed in terms of Fourier transformed propagators. As in the previous two sections, it is instructive to find the vacuum expectation value of the gluon insertion $\Delta^{g}$ with the chiral field strength stemming from the $[i-1 i]$-link and gauge fields, from $[i i+1]$ and $[i+1, i+2]$ in Fig. 3 (a) and (b), respectively. It reads

$$
\Delta_{\alpha \beta}^{\gamma \dot{\gamma}}=\int d^{D} z \mathrm{e}^{i k \cdot z}\left\langle F_{\alpha \beta}\left(x_{1}\right) \Delta^{\mathrm{g}}(z) A^{\gamma \dot{\gamma}}\left(x_{2}\right)\right\rangle
$$

Since $\Delta^{\mathrm{g}}$ is a sum of both chiral and antichiral components of the field strength, one needs the following propagators

$$
\begin{aligned}
\left\langle A_{\alpha \dot{\alpha}}\left(x_{1}\right) F_{\beta \gamma}\left(x_{2}\right)\right\rangle & =\frac{i}{2} \int \frac{d^{D} k}{(2 \pi)^{D}} \frac{\mathrm{e}^{-i k \cdot x_{12}}}{k^{2}} \varepsilon_{\alpha(\beta} k_{\gamma) \dot{\alpha}}, \\
\left\langle F_{\alpha \beta}\left(x_{1}\right) \bar{F}_{\dot{\alpha} \dot{\beta}}\left(x_{2}\right)\right\rangle & =\frac{i}{4} \int \frac{d^{D} k}{(2 \pi)^{D}} \frac{\mathrm{e}^{-i k \cdot x_{12}}}{k^{2}} k_{(\alpha \dot{\alpha}} k_{\beta) \dot{\beta}}, \\
\left\langle F_{\alpha \beta}\left(x_{1}\right) F_{\gamma \delta}\left(x_{2}\right)\right\rangle & =\frac{i}{4} \int \frac{d^{D} k}{(2 \pi)^{D}} \mathrm{e}^{-i k \cdot x_{12}} \varepsilon_{(\alpha \gamma} \varepsilon_{\beta) \delta},
\end{aligned}
$$

where the braces stand for symmetrization of indices $T_{(\alpha \ldots \beta)} \equiv T_{\alpha \ldots \beta}+T_{\beta \ldots \alpha}$. Then, the result of a straightforward calculation, keeping only terms linear in $k$, takes the following compact form

$$
\left.\Delta_{\alpha \beta}^{\gamma \dot{\gamma}}=\frac{i}{4 \pi^{2-\varepsilon}}\left\{\left[\frac{1}{4} x_{12(\alpha}^{\dot{\gamma}} x_{12 \beta) \dot{\delta}} k^{\dot{\delta} \gamma}-k \cdot x_{1} x_{12(\alpha}{ }^{\dot{\gamma}} \delta_{\beta)}\right)^{\gamma}\right] \frac{\Gamma(2-\varepsilon)}{\left[-x_{12}^{2}\right]^{2-\varepsilon}}-\frac{1}{4} k_{(\alpha} \dot{\gamma} \delta_{\beta)} \frac{\Gamma(1-\varepsilon)}{\left[-x_{12}^{2}\right]^{1-\varepsilon}}\right\} .
$$

This can be rewritten in the form that one would get from the use of the covariant Lorentz form of the action, i.e., $F_{\mu \nu}^{2}$. Manipulating the above result, one finds

$\Delta_{\alpha \beta}^{\gamma \dot{\gamma}}=\frac{i}{4 \pi^{2-\varepsilon}}\left\{\left[\frac{1}{4} x_{12}^{\gamma \dot{\gamma}} x_{12(\alpha \dot{\delta}} k_{\beta)}^{\dot{\delta}}-\frac{1}{2} k \cdot\left(x_{1}+x_{2}\right) x_{12(\alpha} \dot{\gamma} \delta_{\beta)}^{\gamma}\right] \frac{\Gamma(2-\varepsilon)}{\left[-x_{12}^{2}\right]^{2-\varepsilon}}-\frac{1}{4} \varepsilon k_{(\alpha}{ }^{\dot{\gamma}} \delta_{\beta)}{ }^{\gamma} \frac{\Gamma(1-\varepsilon)}{\left[-x_{12}^{2}\right]^{1-\varepsilon}}\right\}$.

We observe the presence of $O(\varepsilon)$ effects in this form of writing the expression. These are crucial for getting self-consistent results since the latter contribution induces double poles in $\varepsilon$ in intermediate steps.

The brute force calculation of the diagrams in Fig. 3 is extremely tedious and lengthy. However, the final result is rather compact. The contribution of the graph 3 (a) to the anomaly can be written as

$$
\begin{aligned}
A_{[12][23] ; 1}^{\mathrm{g}}= & a \frac{\Gamma(1-\varepsilon)}{192 \varepsilon}\left(-x_{13}^{2} \mu^{2} \pi\right)^{\varepsilon} \frac{\chi_{1}}{x_{13}^{2}\langle 12\rangle^{2}}\left\{4\langle 12\rangle k \cdot x_{2} \chi_{1} \chi_{2} \eta_{1}+\left(\langle 12\rangle^{3} \eta_{1}^{3}-\chi_{2}^{3}\right)\langle 1|k| 2]\right. \\
& \left.-\langle 12\rangle \chi_{2} \eta_{1}\left(5 \chi_{1}\langle 2|k| 2]-3 \chi_{2}\langle 1|k| 2]\right)+3\langle 12\rangle^{2} \eta_{1}^{2}\left(\chi_{1}\langle 2|k| 2]-\chi_{2}\langle 1|k| 2]\right)\right\}
\end{aligned}
$$

while from (b), it is

$$
\begin{aligned}
& A_{[12][34] ; 1}^{\mathrm{g}}=a \frac{\Gamma(1-\varepsilon)}{192 \varepsilon}\left(-x_{13}^{2} \mu^{2} \pi\right)^{\varepsilon} \frac{\chi_{1} \chi_{2}}{x_{24}^{2} x_{13}^{2}\langle 12\rangle}\left\{-4 k \cdot x_{2} x_{24}^{2} \chi_{1} \eta_{1}+\chi_{1} \chi_{2}[23]\langle 3|k| 1]\right. \\
& \left.\quad+\left(\langle 23\rangle \chi_{1}-\langle 13\rangle \chi_{2}\right)\left[\chi_{2}\left[\frac{[12]}{\langle 12\rangle}\langle 1|k| 3]-\frac{[12]}{\langle 23\rangle}\langle 3|k| 3]\right]+\chi_{1}\left[2 \frac{[23]}{\langle 12\rangle}\langle 2|k| 1]-\frac{[12]}{\langle 12\rangle}\langle 2|k| 3]\right]\right]\right\} .
\end{aligned}
$$


Contrary to their scalar/fermion counterparts, both graphs independently receive contribution which are not translationally invariant, i.e., $\sim\left(k \cdot x_{2}\right)$. However, these do cancel in the sum of the two, echoing the cancellation observed in the case of the anomaly for the bosonic Wilson loop in Section 3.1,

\section{Comments}

Let us comment on the derived expressions. Having found the anomaly in the conformal variation of the super Wilson loop, we can test it against the explicit calculations performed in Section 2. Making use of decomposition of $\eta$ 's in terms of Grassmann components of supertwistors (26) one can extract the $\chi_{1}^{4}$ contribution from Eqs. (70) and (71). Adding to these analogous terms stemming from the mirror symmetric diagrams, one immediately recognizes the anomalous variation of $\langle\mathcal{W}\rangle_{n ; 1}$ displayed in Eq. (41). Actually, one can go in the other direction and predict the anomalous contribution $a_{n ; 1}$ to the super Wilson loop by solving the conformal Ward identity and fixing a possible additive conformally invariant piece by taking the collinear limit to four points. The latter is purely anomalous and was computed at one-loop order for all components of Grassmann degree 4 in Ref. [21]. Along this way we can perform a subtractive transformation on the super Wilson loop and thus restore the conformal symmetry of the object. It is the latter that is dual to non-MHV amplitudes.

Our final comment addresses the question of what components of the super Wilson loop are anomalous and thus require subtractions. An inspection of the right-hand side of the conformal Ward identities immediately suggests that since the only divergent Feynman graphs are those coming from exchanges that involve nearest and next-to-nearest links, the degree 4 Grassmann structure can be anomalous provided it contains at most three adjacent indices, e.g., $\chi_{i-1}^{2} \chi_{i} \chi_{i+1}$, $\chi_{i-1}^{2} \chi_{i}^{2}$ etc. Therefore, any structure where at least one of the indices is not adjacent to the rest will be conformal and given by the corresponding component of the sum of $R$-invariants.

To conclude, the use of one of the most suitable regularization schemes that preserves the helicity-spinor formalism, one the one hand, and allows to tame divergences by means of deviation from the four-dimensional space-time, on the other, inevitably breaks conformal symmetry of the supersymmetric Wilson loop. However the latter, contrary to correlation functions of gauge invariant operators, is a scheme dependent object. It is accompanied by a coefficient function it light-cone OPE. So only the product of the two is expected to be anomaly-free. What we suggested here is to subtract the conformally non-invariant effects that come from the anomalies in the renormalization of the superloop with the variation of the regularized SYM action. The procedure is echoing analogous treatment of mixing effects in the renormalization of conformal operators in gauge theories. This implies that the finite subtraction $a_{n ; 1}$ should compensate the anomalous effects in the OPE coefficient function computed the same order to yield a purely conformal contribution to the correlation function of stress-tensor supermultiplet as was found in Ref. [23].

Several directions are open for further investigation. It is important to understand the allloop structure of the found anomalies. Next, since the consideration of this work was limited to the NMHV level, it necessary to unravel the conformal anomalies for contribution of higher Grassmann degrees. Last but not least, it would be very instructive to define a set of rules on the Lagrangian level that would produce a conformal invariant result after the regularization is removed, thus automatically subtracting the notorious conformal anomalies. 
We would like to thank Gregory Korchemsky for helful correspondence and instructive comments on the manuscript and David Skinner for useful discussions. This work was supported by the U.S. National Science Foundation under grants PHY-0757394 and PHY-1068286.

\section{References}

[1] L. Brink, O. Lindgren, B.E.W. Nilsson, "N=4 Yang-Mills theory on the light cone," Nucl. Phys. B212 (1983) 401;

S. Mandelstam, "Light cone superspace and the ultraviolet finiteness of the $\mathrm{N}=4$ model," Nucl. Phys. B213 (1983) 149.

[2] V.P. Nair, "A current algebra for some gauge theory amplitudes," Phys. Lett. B 214 (1988) 215.

[3] S.J. Parke, T.R. Taylor, "An amplitude for n-gluon scattering," Phys. Rev. Lett. 56 (1986) 2459.

[4] E. Witten, "Perturbative gauge theory as a string theory in twistor space," Commun. Math. Phys. 252 (2004) 189 hep-th/0312171.

[5] J.M. Drummond, J. Henn, G.P. Korchemsky, E. Sokatchev, "Conformal Ward identities for Wilson loops and a test of the duality with gluon amplitudes," Nucl. Phys. B 826 (2010) 337 [arXiv:0712.1223 [hep-th]].

[6] J.M. Drummond, J.M. Henn, "All tree-level amplitudes in N=4 SYM," JHEP 0904 (2009) 018 [arXiv:0808.2475 [hep-th]].

[7] L.J. Mason, D. Skinner, "The complete planar S-matrix of N=4 SYM as a Wilson loop in twistor space," JHEP 1012 (2010) 018 [arXiv:1009.2225 [hep-th]].

[8] S. Caron-Huot, "Notes on the scattering amplitude / Wilson loop duality," JHEP 1107 (2011) 058 [arXiv:1010.1167 [hep-th]].

[9] J.P. Harnad, S. Shnider, "Constraints and field equations for ten-dimensional superYangMills theory," Commun. Math. Phys. 106 (1986) 183;

H. Ooguri, J. Rahmfeld, H. Robins, J. Tannenhauser, "Holography in superspace," JHEP 0007 (2000) 045 hep-th/0007104.

[10] L.F. Alday, J.M. Maldacena, "Gluon scattering amplitudes at strong coupling," JHEP 0706 (2007) 064 arXiv:0705.0303 [hep-th]]; "Comments on gluon scattering amplitudes via AdS/CFT," JHEP 0711 (2007) 068 [arXiv:0710.1060 [hep-th]].

[11] G.P. Korchemsky, J.M. Drummond, E. Sokatchev, "Conformal properties of four-gluon planar amplitudes and Wilson loops," Nucl. Phys. B 795 (2008) 385 [arXiv:0707.0243 [hep-th]].

[12] A. Brandhuber, P. Heslop, G. Travaglini, "MHV amplitudes in N=4 super Yang-Mills and Wilson loops," Nucl. Phys. B794 (2008) 231 [arXiv:0707.1153 [hep-th]]. 
[13] J.M. Drummond, J. Henn, G.P. Korchemsky, E. Sokatchev, "On planar gluon amplitudes/Wilson loops duality," Nucl. Phys. B 795 (2008) 52 arXiv:0709.2368 [hep-th]]; "The hexagon Wilson loop and the BDS ansatz for the six-gluon amplitude," Phys. Lett. B 662 (2008) 456 [arXiv:0712.4138 [hep-th]]; "Hexagon Wilson loop = six-gluon MHV amplitude," Nucl. Phys. B 815 (2009) 142 [arXiv:0803.1466 [hep-th]].

[14] C. Anastasiou, Z. Bern, L.J. Dixon, D.A. Kosower, "Planar amplitudes in maximally supersymmetric Yang-Mills theory," Phys. Rev. Lett. 91 (2003) 251602 arXiv:hep-th/0309040.

[15] Z. Bern, L.J. Dixon, V.A. Smirnov, "Iteration of planar amplitudes in maximally supersymmetric Yang-Mills theory at three loops and beyond," Phys. Rev. D 72 (2005) 085001 arXiv:hep-th/0505205.

[16] C. Anastasiou, A. Brandhuber, P. Heslop, V.V. Khoze, B. Spence, G. Travaglini, "Two-loop polygon Wilson loops in N=4 SYM," JHEP 0905 (2009) 115 [arXiv:0902.2245 [hep-th]].

[17] V. Del Duca, C. Duhr, V.A. Smirnov, "An analytic result for the two-loop hexagon Wilson loop in N = 4 SYM," JHEP 1003 (2010) 099 [arXiv:0911.5332 [hep-ph]]; "The two-loop hexagon Wilson loop in N = 4 SYM," JHEP 1005 (2010) 084 [arXiv:1003.1702 [hep-th]].

[18] A.B. Goncharov, M. Spradlin, C. Vergu, A. Volovich, "Classical polylogarithms for amplitudes and Wilson loops," Phys. Rev. Lett. 105 (2010) 151605 [arXiv:1006.5703 [hep-th]].

[19] Z. Bern, L.J. Dixon, D.A. Kosower, R. Roiban, M. Spradlin, C. Vergu, A. Volovich, "The two-loop six-gluon MHV amplitude in maximally supersymmetric Yang-Mills theory," Phys. Rev. D78 (2008) 045007 [arXiv:0803.1465 [hep-th]].

[20] F. Cachazo, M. Spradlin, A. Volovich, "Leading singularities of the two-loop six-particle MHV amplitude," Phys. Rev. D78 (2008) 105022 [arXiv:0805.4832 [hep-th]].

[21] A.V. Belitsky, G.P. Korchemsky, E. Sokatchev, "Are scattering amplitudes dual to super Wilson loops?," Nucl. Phys. B 855 (2012) 333 [arXiv:1103.3008 [hep-th]].

[22] Z. Bern, A. De Freitas, L.J. Dixon, H.L. Wong, "Supersymmetric regularization, two loop QCD amplitudes and coupling shifts," Phys. Rev. D 66 (2002) 085002 hep-ph/0202271.

[23] B. Eden, P. Heslop, G.P. Korchemsky, E. Sokatchev, "The super-correlator/super-amplitude duality: Part I," arXiv:1103.3714 [hep-th];

B. Eden, P. Heslop, G.P. Korchemsky, E. Sokatchev, "The super-correlator/super-amplitude duality: Part II," arXiv:1103.4353 [hep-th].

[24] B. Eden, G.P. Korchemsky, E. Sokatchev, "From correlation functions to scattering amplitudes," JHEP 1112 (2011) 002, [arXiv:1007.3246 [hep-th]];

L.F. Alday, B. Eden, G.P. Korchemsky, J. Maldacena, E. Sokatchev, "From correlation functions to Wilson loops," JHEP 1109 (2011) 123 [arXiv:1007.3243 [hep-th]].

[25] S. Ferrara, R. Gatto, A.F. Grillo, "Conformal algebra in space-time and operator product expansion," Springer Tracts Mod. Phys. 67 (1973) 1. 
[26] D. Müller, "Conformal constraints and the evolution of the nonsinglet meson distribution amplitude," Phys. Rev. D 49 (1994) 2525;

A.V. Belitsky, D. Müller, "Broken conformal invariance and spectrum of anomalous dimensions in QCD," Nucl. Phys. B 537 (1999) 397 [hep-ph/9804379].

[27] A. Hodges, "Eliminating spurious poles from gauge-theoretic amplitudes," arXiv:0905.1473 [hep-th].

[28] J.M. Drummond, J. Henn, G.P. Korchemsky, E. Sokatchev, "Generalized unitarity for N=4 super-amplitudes," arXiv:0808.0491 [hep-th].

[29] R. Britto, B. Feng, R. Roiban, M. Spradlin, A. Volovich, "All split helicity tree-level gluon amplitudes," Phys. Rev. D 71 (2005) 105017 hep-th/0503198].

[30] R. Britto, F. Cachazo, B. Feng, "New recursion relations for tree amplitudes of gluons," Nucl. Phys. B 715 (2005) 499 [hep-th/0412308|;

R. Britto, F. Cachazo, B. Feng, E. Witten, "Direct proof of tree-level recursion relation in Yang-Mills theory," Phys. Rev. Lett. 94 (2005) 181602 [hep-th/0501052].

[31] M.L. Mangano, S.J. Parke, Z. Xu, "Duality and multigluon scattering," Nucl. Phys. B 298 (1988) 653. 